\title{
Complete blood count profiles in children with eczema herpeticum
}

\author{
Grace Chan ${ }^{1}$, Choo Phei Wee ${ }^{2}$, and Peck Ong ${ }^{3}$ \\ ${ }^{1}$ Children's Hospital Los Angeles Department of Pediatrics \\ ${ }^{2}$ University of Southern California \\ ${ }^{3}$ Childrens Hospital Los Angeles/USC Keck School of Medicine
}

May 11, 2021

\section{Complete blood count profiles in children with eczema herpeticum}

To the Editor,

Eczema herpeticum (EH) is a significant infectious complication of atopic dermatitis (AD) and may lead to serious problems including keratitis, viremia and meningitis (1). The clinical diagnosis of EH may be challenging as the morphology of EH may be mistaken for $\mathrm{AD}$ exacerbation and bacterial infection (2). Difficulty in diagnosis may impact treatment decisions while confirmatory testing for herpes simplex virus (HSV) is pending.

Previous studies have investigated the association between EH and common laboratory values but have largely been inconclusive. One study in young adults found EH associated with lymphopenia and elevated IgE (3). Another study in children found no relationship between EH and serum total IgE, eosinophil count, or vitamin D level (4).

A complete blood count (CBC) is a common test often obtained when there is concern for more severe infection. In this study, we investigated whether CBC profiles can be utilized to distinguish hospitalized children with $\mathrm{EH}$ from $\mathrm{AD}$ patients with bacterial infections or $\mathrm{AD}$ exacerbation.

Electronic medical records were reviewed for patients 18 years and younger hospitalized with a primary diagnosis of AD between 2003 to 2018 using International Classification of Diseases -9 and -10 codes (5). The study has been approved by the Institution Review Board at Children's Hospital Los Angeles. Patients were sorted into the EH group if there was a positive HSV polymerase chain reaction (PCR) swab from skin lesions. Subjects who had bacterial infections or AD exacerbation were classified based on clinical history, physical examination findings and discharge diagnosis as previously described (5). Briefly, patients with a discharge diagnosis of cellulitis, skin abscess, bacteremia, osteomyelitis, septic arthritis or endocarditis were classified into bacterial infections whereas those with generalized, severe eczema exacerbation with documented outpatient treatment failure were classified into AD exacerbation. A CBC obtained within 48 hours of admission was utilized. Multivariate linear regression model was used to assess the effect of EH on the CBC profile adjusting for patient age and gender. Statistical significance was set at $5 \%$ level with two-sided test throughout the analysis. All statistical computations were done by Stata/SE 16.0 (StataCorp, College Station, TX).

130 subjects with an admission CBC were included in the study. Twenty-two (17\%) had EH based on positive HSV PCR. Forty-nine (38\%) had AD exacerbation while fifty-nine (45\%) had bacterial infectious complications such as cellulitis, skin abscess, bacteremia, osteomyelitis or septic arthritis.

EH patients had significantly lower mean admission white blood cell count (WBC, $9.7 \pm 6.4$ vs. $15.7 \pm 9.1$ vs $15.9 \pm 6.8 ; p=<0.0001$ ), absolute neutrophil count (ANC, $4.9 \pm 5.0$ vs. $5.7 \pm 4.7$ vs. $9.0 \pm 5.6 ; p=0.01$ ), and absolute lymphocyte count (ALC, $3.5 \pm 1.8$ vs. $6.4 \pm 4.6$ vs. $4.9 \pm 3.4 ; p=0.003$ ), as compared to AD 
exacerbation and bacterial infection, respectively (Table 1). When compared to published reference ranges (6), $13.6 \%$ of those with $\mathrm{EH}, 2 \%$ of those with $\mathrm{AD}$ exacerbation, and $0 \%$ of those with bacterial infection had absolute leukopenia for age (WBC under lower limit of normal) (Table 2A). $4.5 \%$ of those with EH, $6.1 \%$ of those with $\mathrm{AD}$ exacerbation, and $0 \%$ of those with bacterial infection had absolute neutropenia for age (Table 2B). $22.7 \%$ of patients with EH demonstrated absolute lymphopenia for age (Table 2C). In comparison, $10.2 \%$ of those with $\mathrm{AD}$ exacerbation and $13.6 \%$ of those with bacterial infection had absolute lymphopenia for age.

$\mathrm{EH}$ is a skin infection caused by HSV that can lead to serious complications including ocular and systemic infections. Therefore, accurate diagnosis is important in determining appropriate treatments. The initiation of acyclovir for EH is based on clinical impression, as test results such as PCR and culture are not immediately available. Our current findings may have clinical implications for treatment when CBC profiles are taken together with physical examination as EH may be difficult to distinguish from some bacterial skin infections. For example, streptococcal pustulosis may present with punched scalloped borders that mimic EH (2).

Large studies of EH in children are rare. Our results corroborate Wollenberg et al 's finding that lymphopenia is more pronounced in patients with EH (3). Lymphopenia in the setting of a normal white blood cell count in EH patients may be a finding related to HSV infection, as another article studying the effect of HSV infection (not specifically EH) on CBC parameters also found lower lymphocyte counts in those with HSV (7).

In conclusion, our results show that leukopenia or lymphopenia in light of clinical suspicion of EH may help clinicians in their treatment decision. Our study has limitation in that it is based on hospitalized patients and may not apply to an outpatient setting.

Keywords: acyclovir, eczema herpeticum, CBC, lymphopenia, leukopenia, neutropenia

Grace Chan, MD ${ }^{\mathrm{a}}$, Choo Phei Wee, MS ${ }^{\mathrm{b}}$, Peck Y. Ong, MD ${ }^{\mathrm{c}, \mathrm{d}}$

${ }^{a}$ Division of General Pediatrics, Children's Hospital Los Angeles

bBiostatistics core, The Saban Research Institute, Children's Hospital Los Angeles, and Southern California and Clinical Translation Science Institute, University of Southern California.

${ }^{\mathrm{c}}$ Division of Clinical Immunology and Allergy, Children's Hospital Los Angeles.

${ }^{\mathrm{d}}$ Keck School of Medicine, University of Southern California, Los Angeles, California

This study was funded in part by a Merit Award from The Saban Research Institute at Children's Hospital Los Angeles to PYO. CPW is supported in part by NIH/NCRR SC-CTSI Grant UL1 TR000130 (Biostatistics core, CHLA/USC).

The authors have no conflicts of interest to declare.

Address correspondence to: Peck Y. Ong, MD, Division of Clinical Immunology and Allergy, Children's Hospital Los Angeles, 4650, Sunset Blvd, MS\# 75, Los Angeles, CA 90027. Tel: 323-361-2501. Email: pyong@chla.usc.edu

\section{References}

1. Sun D, Ong PY. Infectious Complications in Atopic Dermatitis. Immunol Allergy Clin North Am 2017; 37(1):75-93. doi:10.1016/j.iac.2016.08.015

2. Shayegan LH, Richards LE, Morel KD, Levin LE. Punched-out erosions with scalloped borders: Group A Streptococcal pustulosis. Pediatr Dermatol 2019; 36: 995-996. doi:10.1111/pde.13956

3. Wollenberg A, Zoch C, Wetzel S, Plewig G, Przybilla B. Predisposing factors and clinical features of eczema herpeticum: a retrospective analysis of 100 cases. J Am Acad Dermatol. 2003;49(2):198-205. doi:10.1067/s0190-9622(03)00896-x 
4. Kim K, Kang J, Won Kim S, Sung M. Relationship between the presence of eczema herpeticum and the significance of clinical and laboratory tests in Korean children with atopic dermatitis. Iran J Pediatr. 2016;26(4):e4683. doi:10.5812/ijp.4683

5. Wang V, Keefer M, Ong PY. Antibiotic choice and methicillin-resistantStaphylococcus aureus rate in children hospitalized for atopic dermatitis. Ann Allergy Asthma Immunol 2019;122(3):314-317. doi:10.1016/j.anai.2018.12.001

6. Lubin BH. Reference values in infancy and childhood. In: Nathan DG, Oski FA, editors. Hematology of Infancy and Childhood. 2nd edition. Philadelphia, Pa, USA: WB Saunders; 1981. pp. 1552-1574. 10.1016/j.jaci.2003.07.003.

7. Asaduzzaman M, Mrida AH, Hasan N, et al. The effect of herpes simplex virus infection on different blood parameters: A transverse study. IOSR-JDMS 2018;17(9):81-89.

\section{Hosted file}

Tables Final.pdf available at https://authorea.com/users/413175/articles/521579-completeblood-count-profiles-in-children-with-eczema-herpeticum 